\title{
Morphological, chemical and thermal analysis of cellulose nanocrystals extracted from bamboo fibre
}

\begin{abstract}
This work investigates the extraction of cellulose nanocrystals (CNC) from bamboo fibre as an alternative approach to utilize the waste bamboo fibre. In this study, bamboo fibre was subjected to acid hydrolysis for efficient isolation of CNC from bamboo fibre. The extracted CNC's were morphologically, characterized via Scanning Electron Microscopy (SEM), Transmission Electron Microscopy (TEM) and Atomic Force Microscopy (AFM). The energy Dispersive X-rays (EDX) provided the elemental composition of the prepared CNC's and Xray diffractometer (XRD) exhibited their crystallinity. The physiochemical analysis was done via Fourier Transform Infrared (FTIR); and their thermal analysis was revealed by Thermogravimetric Analysis (TGA) and Differential scanning calorimetry (DSC). As from their morphological investigations, rod like structures of CNC's were observed under SEM analysis with higher carbon content as demonstrated by EDX, while needle shaped CNC's were observed from TEM and AFM studies. Acid hydrolysis for 45 min resulted into higher degree of crystallinity and higher yield of CNC's about $86.96 \%$ and $22 \%$ respectively. Owing to higher quality of CNC's obtained as a result of efficient and modified techniques, these can find potential usage in nanocomposites for biomedical and food packaging application.
\end{abstract}

Keyword: Nano crystalline cellulose; Bamboo pulp; Morphological properties; Thermal properties; Chemical properties 